\title{
Analysis of Data from the Low-energy Modes of the Surface Detector of the Pierre Auger Observatory
}

\author{
Martin Schimassek ${ }^{* a}$ for the Pierre Auger Collaboration ${ }^{\dagger b}$ \\ ${ }^{a}$ Karlsruhe Institute of Technology (KIT), ETP, Germany \\ ${ }^{b}$ Observatorio Pierre Auger, Av. San Martín Norte 304, 5613 Malargüe, Argentina \\ E-mail: auger_spokespersons@fnal.gov \\ Full author list: http://www.auger.org/archive/authors_icrc_2019.html
}

\begin{abstract}
The Pierre Auger Observatory is the largest cosmic-ray observatory in existence and covers an area of about $3000 \mathrm{~km}^{2}$ with its 1660 surface detectors. Each of these detector stations records signals of relativistic particles in 12 tonnes of ultra-pure water, measured with three 9-inch photomultipliers. With a collecting area of about $10 \mathrm{~m}^{2}$, each surface detector station is sensitive to single particles down to $\mathrm{MeV}$ energies. Such particles are recorded in counters and histograms for each detector and transmitted to the central data acquisition system at regular time intervals. The background flux of particles is measured with very high statistics by combining all surface detector stations and therefore enables investigations of the source of variations in this flux. We present an updated analysis of the particle-counter data and highlight the long-term stability of the corrected rates. With these corrected rates, we present the frequency spectrum of the observed variations and highlight the expected solar contributions, show an in-depth analysis of the diurnal variation, and update the observation of the eleven-year solar-cycle from 2006 until the end of 2018.
\end{abstract}

36th International Cosmic Ray Conference - ICRC2019

24 July - 1 August, 2019

Madison, Wisconsin, USA

\footnotetext{
${ }^{*}$ Speaker.

${ }^{\dagger}$ for collaboration list see PoS(ICRC2019)1177
} 


\section{Introduction}

The rate of cosmic rays measured at the top of Earth's atmosphere is modulated by changes in the interplanetary medium. Solar activity and transient events modify the magnetic deflections of high-energy particles and thus change the cosmic ray intensity at Earth.

The standard detectors for measuring this intensity are neutron monitors, that measure the hadronic component of the particle cascades formed in Earth's atmosphere. However, also different detector systems are able to track the evolution of the cosmic-ray intensity, for example muon telescopes and particle counters from high-energy experiments.

The Pierre Auger Observatory [1] is designed to measure the highest energy cosmic rays. It is located in Malarguie, Argentina $\left(69.3^{\circ} \mathrm{W}, 35.3^{\circ} \mathrm{S}, 1400 \mathrm{~m}\right.$ a.s.1.) and covers $3000 \mathrm{~km}^{2}$ with its surface detector (SD). The surface detector is built as a triangular grid with $1500 \mathrm{~m}$ spacing of 1660 water-Cherenkov detector stations. Each of these detector stations is constantly measuring the rate of particles for stability monitoring and calibration $[1,2]$.

This data has been previously employed in studies of solar transient events like Forbush decreases [2, 3, 4], searches for indications of gamma-ray bursts [5], and identification of modulations related to the solar cycle [6].

In detail, the rate of particles in the SD stations is measured using a counting trigger, the socalled scaler mode, with a very low current threshold range of $3<I_{\text {peak }}<21$ ADC channels above baseline [2]. A coincidence of all 3 PMTs of the station is required. This counter is sensitive to a deposition of energy in the range $15 \mathrm{MeV} \lesssim E_{\mathrm{d}} \lesssim 100 \mathrm{MeV}$ [3] and results in an average count rate of $\sim 1.8 \times 10^{8}$ counts per minute, where the rate is reported every second via a radio transmission. This energy depositions arise from showers from primary cosmic-rays with energies from $10 \mathrm{GeV}$ to a few $\mathrm{TeV}$ [3]. A different source of data are pulse-height and pulse-charge histograms collected for calibration purposes, and are available together with the high-energy cosmic ray events. These can also be used to study the background intensity of particles [6].

In this work, we focus on the scaler data and present an updated analysis of the existing data set. In Section 2 we present the detection of lightning strikes using the scaler mode. The updated data treatment is discussed in Section 3. In the following Sections we present a first spectral analysis of the corrected scaler data, a detailed analysis of the diurnal modulation, as well as updated analyses on the modulations during Forbush decreases and during the solar cycle, with data covering 13 years.

\section{Lightning Events}

Since the implemented threshold is low, the scaler rate is sensitive to the electromagnetic pulse associated with lightning strikes. Identifying seconds with lightning strikes is thus important for background rejection in analyses of the scaler rate. In addition, other analyses, carried out with the Auger SD, can profit from the identification power for these events.

The general concept for identifying seconds with lightning strikes is similar to the search of rate increase correlated with gamma-ray bursts [5]. By comparing the scaler rate measured in the test second $\Gamma\left(t_{i}\right)$ with the mean rate recorded in a five minute interval $\langle\Gamma\rangle$, significant excesses are selected. In this analysis we take the median of 300 -second intervals as a robust estimate of the 

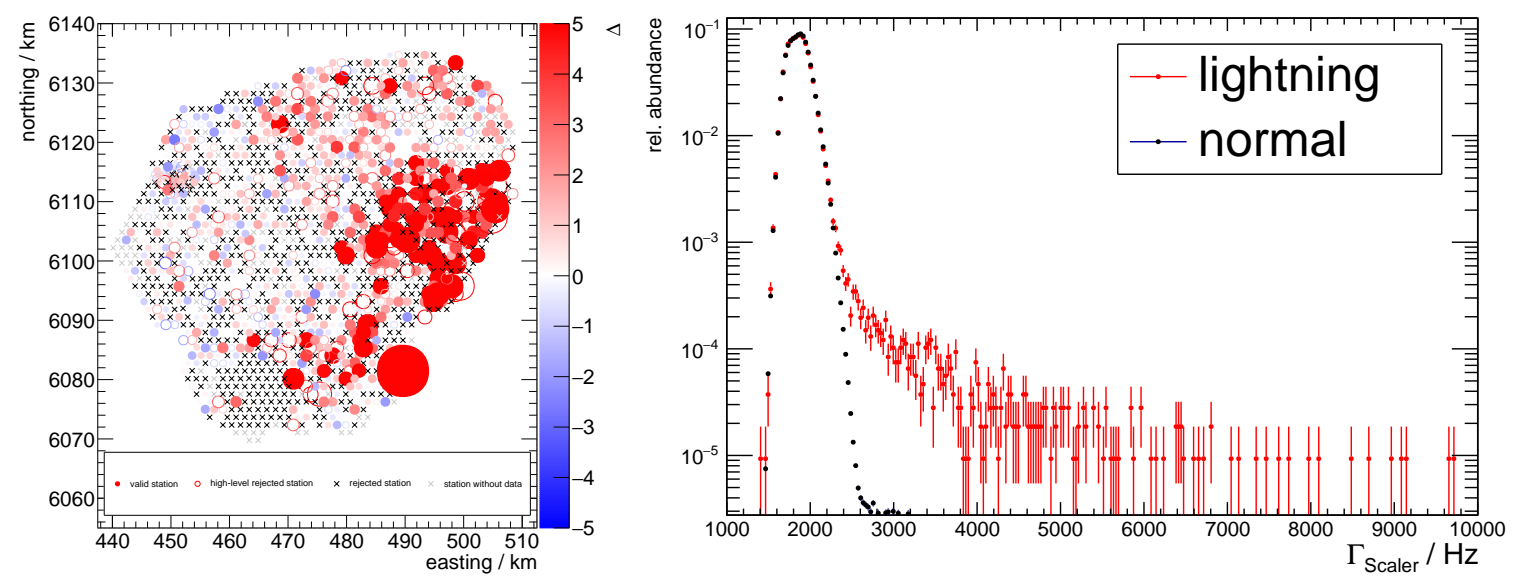

Figure 1: Left: Visualization of a lightning-strike event. The color and sizes of the markers represent the deviation of the measured scaler rate from the median rate within a 5-min interval. See text for more details. Right: Distribution of measured scaler rates during seconds including lightning strikes (red) and during normal conditions (black).

mean rate $\langle\Gamma\rangle$, and estimate the current standard deviation $\hat{\sigma}$ with the median absolute deviation (MAD) under a Gaussian assumption. These quantities are defined for each available SD station separately.

We use a Gaussian background model with a common additional signal $s$ to estimate the significance of excesses. With the rates of all stations $\vec{\Gamma}$, their respective median rates $\langle\vec{\Gamma}\rangle$, and the estimated standard deviations $\overrightarrow{\hat{\sigma}}$, the likelihood of an additional excess signal is

$$
\mathscr{L}(\vec{\Gamma} \mid\langle\vec{\Gamma}\rangle, \overrightarrow{\hat{\sigma}}, s)=\prod_{i} \frac{1}{\sqrt{2 \pi} \sigma_{i}} \exp \left[-\left(\frac{\Gamma_{i}-\langle\Gamma\rangle_{i}-s}{\sqrt{2} \sigma_{i}}\right)^{2}\right] .
$$

After maximising with respect to $s$, Wilk's theorem is used to estimate its significance. We discriminate lightning seconds from other increases using the fit-quality of selected significant excess seconds. When the excess is originating from a lightning strike, the SD stations with a high signal are few and localised so that the quantity

$$
\chi^{2}=\sum_{i} \frac{\left(\Gamma_{i}-\langle\Gamma\rangle_{i}-\hat{s}\right)^{2}}{\sigma_{i}^{2}+\hat{s}}
$$

where $\hat{s}$ is the estimated common signal, is very large due to the rest of the SD array showing no excess. Seconds with lightning strikes can be identified by using the Gaussian transformation of the goodness-of-fit $\chi^{2}$ with the criterion $\left(\chi^{2}-N_{\text {DoF }}\right) / \sqrt{2 N_{\text {DoF }}} \gtrsim 100$.

For a lightning-strike event the pull $\Delta=(\Gamma-\langle\Gamma\rangle) / \hat{\sigma}$ of each station is visualised in the left panel of Fig. 1. A localised excess is clearly visible in the eastern part of the SD. Stations that are not used in the analysis of the scaler data are drawn with crosses and the criteria for their selection is described in the next Section.

By stacking all identified seconds with lightning strikes during the single day 24 January 2016, as well as the "quiet" seconds, the difference in the distribution of the scaler counts during lightning strikes is made visible. In the right panel of Fig. 1, histograms for this example day are shown. A 
clear deviation from the Gaussian distribution during lightning periods is visible. The seconds identified as affected by lightning strikes are not used in the analyses presented in the following Sections.

\section{Data Treatment}

We have shown in previous work $[2,6]$ that the scaler data has to be handled carefully due to the low threshold employed. Additionally, for its interpretation over longer time scales $(\gtrsim$ 5 minutes), corrections have to be applied $[4,6]$.

Here we present an update of the data treatment presented in [6]. This update is based on a change of a paradigm: To recover more data, all selections and corrections are performed on the single-station level. To ensure good data quality, the PMT-status information from the analysis of air-shower traces [7] is used to select stations with three well working PMTs. This selection replaces also cuts based purely on the extrema of the scaler-rate distribution.

Similarly to the previous analyses, the atmospheric overburden altering the rate of measurable particles, is then corrected by the means of a linear fit of the pressure dependency. Under the assumption that the count rate scales with the pressure difference $\Delta P=P-\langle P\rangle$, we use the fit function

$$
\Gamma=\left(1+\alpha_{P} \Delta P\right) \Gamma_{0}
$$

with now station-specific constants $\alpha_{P}$ and $\Gamma_{0}$. The final pressure correction is then in a form of a scaling factor $\gamma_{P}(t)=1-\alpha_{P} \Delta P$ available for each SD station.

As we have shown in [6], the changes in the optical properties of the water-Cherenkov detectors influence the detection threshold of low-energy particles over longer time periods of several years [8]. To measure these optical properties of an SD station we use the so-called "area-overpeak" quantity: the measured charge of a muon-produced pulse, divided by its height. This quantity is computed for each PMT of a SD station and the average per station is used for this correction. We use it to calculate a time dependent correction factor $\gamma_{\mathrm{AoP}}(t)$ for each station, similar to the procedure in [6].

Using both corrections, the corrected scaler rate $\hat{\Gamma}$ of station $i$ is calculated as

$$
\hat{\Gamma}_{i}(t)=\Gamma_{i}(t) \gamma_{P}(t) \gamma_{\mathrm{AoP}}(t) \text {. }
$$

To account for differences in the SD stations, as for example different altitudes, we will use in the following a relative quantity based on the scaled count-rates of the stations. For each station a reference rate $\Gamma_{0}^{(i)}$ is calculated as its mean rate during the year 2013. We then use the average of these rescaled rates

$$
r_{\text {scaler }}(t)=\frac{1}{n} \sum_{i} \hat{\Gamma}_{i}(t) / \Gamma_{0}^{(i)}
$$

averaged over five-minute intervals as basic scaler data quantity.

\section{Observed Frequency Spectrum}

As pointed out in the introduction, the intensity of cosmic-ray with energies of about $\lesssim$ $100 \mathrm{GeV}$ measured at Earth is modulated by solar effects. In this analysis we want to discuss the periodic parts of this modulation as observed in the Auger scaler rate. 

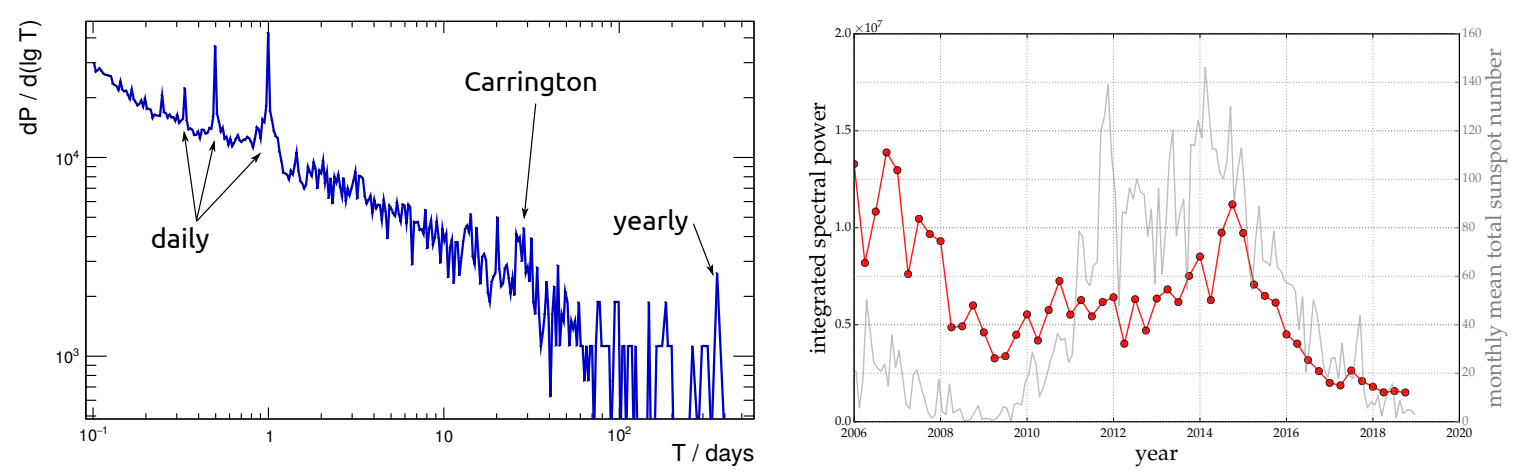

Figure 2: Left: Observed frequency spectrum over the full data period from 2006 to 2018. Right: Time dependence of the integrated spectral power around the Carrington frequency $T \in[20,70]$ days including overtones. For reference, the solar sunspot-number data from [9] is shown in gray.

The left panel of Fig. 2 shows the discrete Fourier-series expansion of the observed scaler rates in logarithmic scale. The sharpest peaks in the spectral power are visible for a signal with a frequency of one solar day and the corresponding overtones. This diurnal signal is due to the Earth's rotation in the magnetized solar wind and has been observed with Auger before [4]. A more detailed analysis of this signal is presented in the next section.

Another expected periodicity of the cosmic-ray intensity is associated with the rotation of the Sun and corresponding magnetic features. Therefore, an enhancement in the spectrum at frequencies corresponding to the Carrington period is expected. On the logarithmic scale of Fig. 2 this is not clearly visible. However, small enhancements at the indicated range are visible and the frequency spectrum is not expected to show a clear peak at the Carrington frequency, because of the different frequencies of individual transient events contributing.

Because the rate of magnetic features of the Sun correlates with the solar cycle, a time dependence of the spectral power of these frequencies is expected. The right panel of Fig. 2 shows the integrated spectral-power for Fourier modes with periods between 20 and 70 days. For reference, the number of sunspots is shown during the same period as well. The minima and the solar maxima in sunspot number also appear as minima and maximum of the spectral power. But, as expected from the presence of non-periodic solar events like Forbush decreases, there is no one-to-one correlation of the spectral power with the sunspot number.

In the long-period domain an enhancement of the Fourier modes with a period of 365 days is visible, corresponding to the movement of the Earth around the Sun. The 11-year solar cycle cannot be resolved with this Fourier analysis and the length of the data set of only 13 years. A time correlation based approach for this observation is presented in the last Section of this work.

\section{Diurnal Signal}

The rotation of the Earth in the magnetized solar wind gives rise to a diurnal change in the measured rate of particles at the ground. Due to the tilt of the Earth's rotation axis and the location of Auger at about $35^{\circ}$ south latitude, as well as the minor changes in distance to the Sun, yearly modulations of this signal are also expected. 
The data set of the corrected Auger scalers show these modulations that are not correlated with pressure or temperature very clearly. The left panel of Fig. 3 shows these daily modulations of the measured scaler rates. For each month the Auger scaler rates are averaged in five-minute bins in time of day (UTC) and rescaled with the average scaler rate in this month. This rescaling emphasizes the shape of the diurnal signal, with an amplitude of about 1\%, instead of highlighting the long-term variations caused by the 11-year solar-cycle which is measured with an amplitude of about $4 \%$.

From Fig. 3 the seasonal variation of the diurnal signal is clearly visible. The peak position of the signal is shifting by three hours over the course of a year. In January the daily signal peaks at about 14:00 UTC, whereas in austral winter the peak moves to 17:00 UTC.

During the solar maximum in the years from 2012 to 2014 the daily signals are less similar. In this period the regular structure is washed out by a higher rate of transient events and higher variability.

The long-term stability of the daily profiles can be derived from comparing the daily profiles across the 13 years shown in Fig. 3. Apart from resolution effects from the SD still being deployed during 2006, the profiles of 2006 match those measured in 2018.

With the high statistics available at the Auger, the diurnal modulation of on average 1.2\% peakto-peak is resolved every day. This is also the reason for the strong enhancement of the spectral power for frequencies of one per day and its overtones in the Fourier spectrum, shown in the left panel of Fig. 2.

In the right panel of Fig. 3 this ability to resolve the signal is visualized by comparing the average profile of June 2018, drawn in black, with the profiles of two individual days of this month.

The data of the individual days are rescaled with their average rate, to enable the comparison of the shapes themselves. For both chosen days the diurnal variation is clearly visible. However, the amplitudes and details of the signals are distinctively different: the peak-to-peak amplitude for June 1 st with $0.7 \%$ is smaller than in the average profile, which shows an amplitude of $1.4 \%$. In contrast to June 1st, the peak-to-peak amplitude of June 5th is very similar to the average profile, but this day also shows that with the high statistics of the Auger scaler rate, other features, only present in singular days, are resolved. In this example of June 5th there is an additional rate enhancement at about 8:00 UTC, as well as two additional depressions of the rate at 2:00 and 6:00 UTC.

This comparison highlights the abilities of the Auger scaler rates in resolving structures on hourly scales with high statistics. This is the basis for future analysis of the rates on these scales also during transient events like Forbush decreases.

\section{Observation of Forbush Decreases}

The time resolution of the Auger scaler rate of up to $1 \mathrm{~s}$ can be used to search for transient events. One example of transient solar events are Forbush decreases. After applying the pressure correction the Auger scaler rates are sensitive to Forbush decreases and they have been observed and analysed before [3].

With this updated analysis and corrections, these observations are possible as well and profit from the updated corrections. In the right panel of Fig. 4 an example of a Forbush decrease, as observed with this new Auger scaler data set, is shown. For comparison, data from three different 

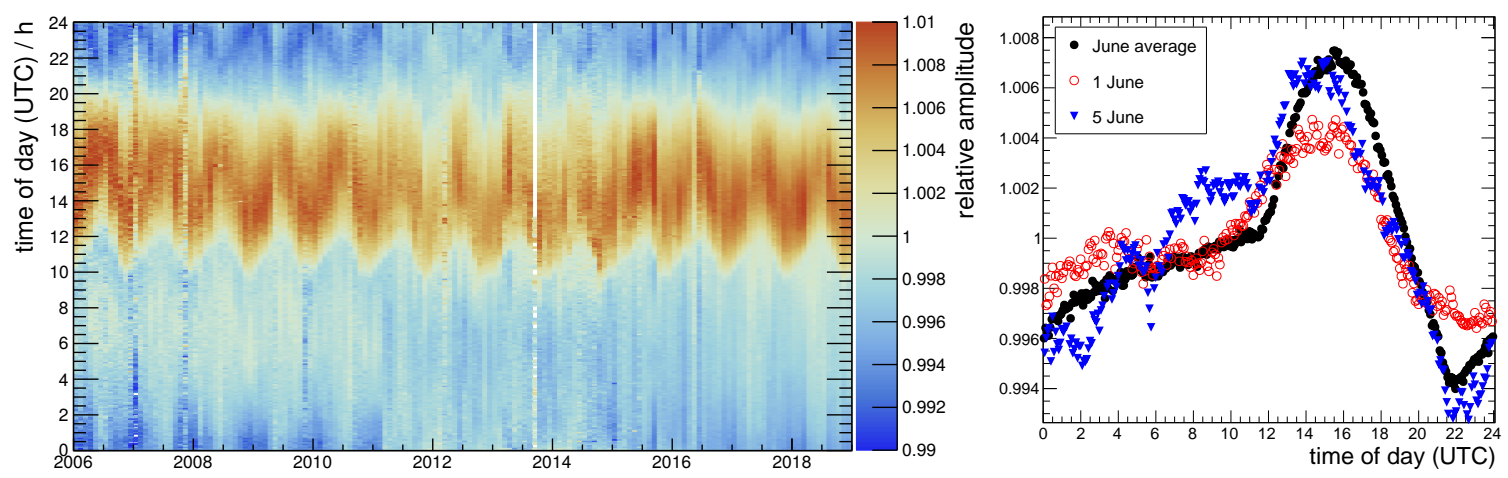

Figure 3: The observed daily pattern of the scaler rate as a function of the time of day (UTC). Left: The monthly average values. Each month is normalized separately to emphasize the shape. Right: For June 2018: the average daily shape of the observed diurnal pattern together with two single days as examples of the variations in this diurnal pattern.

neutron monitors [10], Athens [12] in the northern hemisphere, McMurdo [13] in the Antarctic, and Tsumeb in Namibia [11] are shown for the same time period. The Forbush decrease mid of June 2015 is clearly visible in all stations, with the Auger scaler rate showing the smallest relative amplitude due to the high magnetic cut-off.

Besides the Forbush decrease itself, there are more features in the neutron-monitor time-series which can also be observed in the Auger scaler rate. One example is the enhancement in the rates during the end of July 2015.

This shows the potential of the Auger scaler rate to complement neutron-monitor data at highrigidity cut-off in the southern hemisphere.

\section{Long Term Observations}

The Auger scaler rate can be used for long-term measurements of solar modulations of the cosmic-ray intensity as it was shown in previous work [6]. To achieve this long term stability of the measurements, the corrections, as presented in [6] and their adaptation to a station-based approach in Eq. (3.2), are essential. Here we present an updated version of the 11-year solar-cycle modulation of the cosmic-ray intensity. The left panel of Fig. 4 displays the measured scaler rate from January 2006 until the end of 2018. For comparison, the rate as measured by the Tsumeb neutron monitor [11] is shown during the same time period.

Over the whole period we observe a variation in the average scaler rate of about $4 \%$. With a maximum of $+3 \%$ relative to Jan 2006 in 2009 and a minimum with $-1 \%$ in 2015. In June 2018 the amplitude reaches again about $+3 \%$ relative to Jan 2006 during the current solar minimum. The correlation with the neutron monitor - as well as the match with the expected behaviour from solar sunspot data [9] - showing a recovery of the scaler rate towards the second solar minimum during the operation of Auger, highlight the long-term stability and reliability of the corrected scaler rate.

\section{Conclusion and Outlook}

We have shown that with the appropriate corrections the Auger Scaler mode is a useful data set 

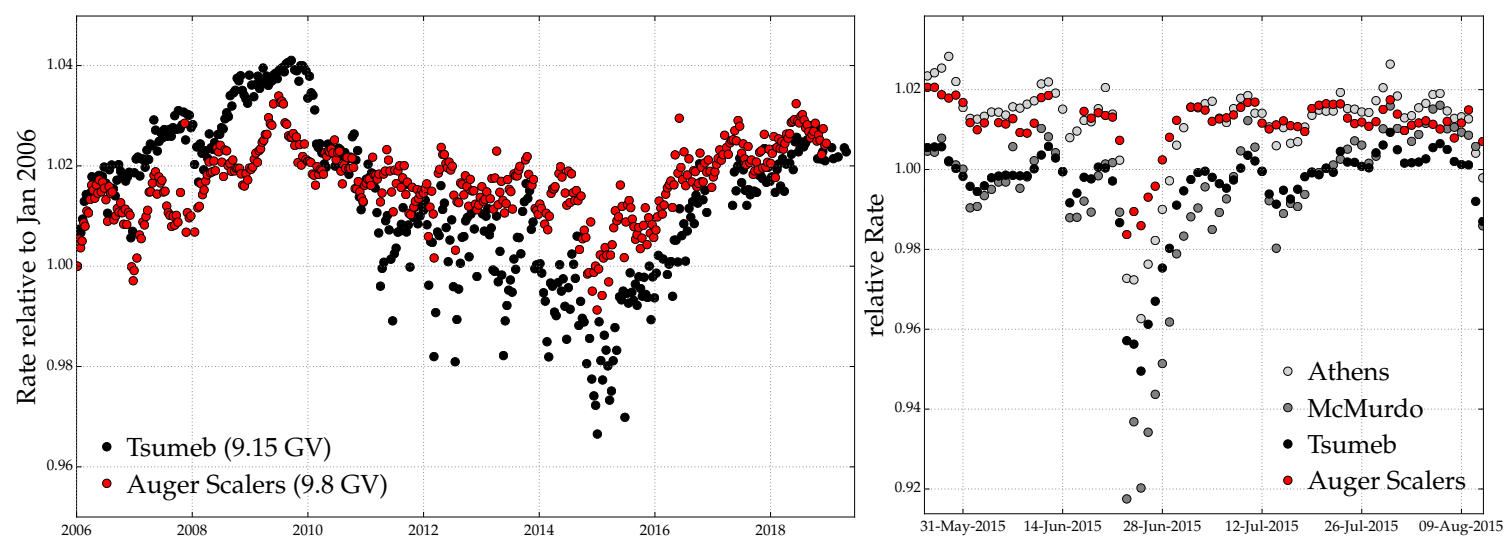

Figure 4: Left: Long-term comparison of the measured rates of the Tsumeb [11] neutron monitor with the corrected Auger scaler rate. Data from 2006 until the end of 2018 is shown, averaged in 10 day intervals. Right: Comparison of the measured Auger scaler rates during a Forbush decrease with data from the Athens [12], McMurdo [13], and Tsumeb [11] neutron monitors.

to measure modulations of the cosmic-ray intensity at time scales from hours to several years. The good correlation of the long-term measurements with neutron-monitor data highlights the validity of our corrections. An additional proof of the stability of our data and the corrections is the stability of the phase and amplitude of the diurnal variation in the measured rate over the range of 13 years.

In the near future there is also the possibility to extend the set of low-energy data collected with Auger because of the new possibilities introduced with the upgraded detectors [14]. With new electronics [15] and a complementary type of particle detector, new possibilities to measure the total cosmic-ray intensity will be explored.

\section{References}

[1] A. Aab [Pierre Auger Collaboration], Nucl. Instrum. Meth. A, 798 (2015) 172.

[2] The Pierre Auger collaboration, JINST, 6 (2011) P01003, doi: 10.1088/1748-0221/6/01/P01003.

[3] S. Dasso, H. Asorey et al. [Pierre Auger Collaboration], Adv. Space Res., 49 (2012) 1563-1569, doi: 10.1016/j.asr.2011.12.028.

[4] H. Asorey [Pierre Auger Collaboration], 31st Int. Cosmic Ray Conf., Łódź, Poland (2009), 41.

[5] X. Bertou [Pierre Auger Collaboration], ICRC (2007), 1042.

[6] J.J. Masias-Meza [Pierre Auger Collaboration], PoS(ICRC 2015) 074, arXiv: 1509.03732.

[7] D. Mockler [Pierre Auger Collaboration], PoS(ICRC2019)353.

[8] K. Choi [Pierre Auger Collaboration], PoS(ICRC2019)222.

[9] SILSO World Data Center, International Sunspot Number Monthly Bulletin and online catalogue, 2006-2018.

[10] The NMDB database (www.nmdb.eu), founded under the European Union's FP7 programme provided the data.

[11] TSUMEB Neutron Monitor, North-West University of South Africa. Data accessed via NMDB.eu.

[12] H. Mavromichalaki et al., Ann. Geophys., 23 (2005) 1-8.

[13] Data kindly provided by the Univ. of Delaware Dep. of Physics and Astronomy and the Bartol Research Institute.

[14] A. Aab et al. [Pierre Auger Collaboration], arXiv: 1604.03637.

[15] D. Nitz [Pierre Auger Collaboration], PoS(ICRC2019)370. 